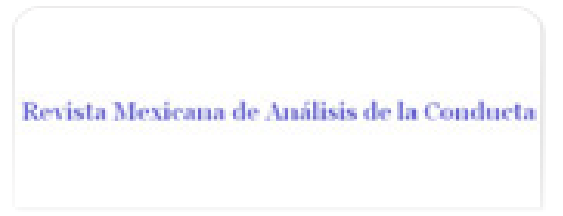

Revista Mexicana de Análisis de la Conducta ISSN: 0185-4534

editora@rmac-mx.org

Sociedad Mexicana de Análisis de la Conducta México

López, Christian; Bruner, Carlos A.

La formación de una discriminación operante en una situación de beber inducido por el programa

Revista Mexicana de Análisis de la Conducta, vol. 33, núm. 2, 2007, pp. 99-117

Sociedad Mexicana de Análisis de la Conducta

Guadalajara, México

Disponible en: http://www.redalyc.org/articulo.oa?id=59333201

- Cómo citar el artículo

- Número completo

- Más información del artículo

- Página de la revista en redalyc.org

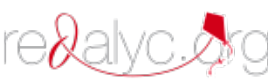

Sistema de Información Científica

Red de Revistas Científicas de América Latina, el Caribe, España y Portugal Proyecto académico sin fines de lucro, desarrollado bajo la iniciativa de acceso abierto 


\title{
LA FORMACIÓN DE UNA DISCRIMINACIÓN OPERANTE EN UNA SITUACIÓN DE BEBER INDUCIDO POR EL PROGRAMA
}

\author{
THE FORMATION OF AN OPERANT DISCRIMINATION \\ IN A SCHEDULE-INDUCED DRINKING SITUATION \\ CHRISTIAN LÓPEZ Y CARLOS A. BRUNER ${ }^{1}$ \\ UNIVERSIDAD NACIONAL AUTÓNOMA DE MÉXICO
}

\begin{abstract}
RESUMEN
Los intentos previos por condicionar el beber inducido por el programa (BIP) a un estímulo previamente neutral involucraron procedimientos de condicionamiento Pavloviano. Esta estrategia produjo resultados mixtos. De acuerdo con la idea de que es posible reducir el BIP a un caso de condicionamiento operante en el que se refuerza con agua a la respuesta que la produce, se intentó establecer el control de estímulos sobre el BIP usando un procedimiento de discriminación operante. Se expuso a tres ratas privadas de comida a programas mixtos y múltiples de reforzamiento con agua. En componentes de $32 \mathrm{~s}$ las presiones a una palanca produjeron agua conforme a un programa de reforzamiento de intervalo al azar $6 \mathrm{~s}$. En componentes de $64 \mathrm{~s}$ las presiones a la palanca no produjeron reforzamiento con agua. Concurrentemente se entregó comida conforme a un programa de tiempo al azar $64 \mathrm{~s}$. Para las tres ratas, la tasa de respuesta fue similar en los dos componentes cuando se mantuvieron en efecto los programas mixtos. También para las tres ratas,
\end{abstract}

1. El presente estudio formó parte de la tesis doctoral del primer autor, cuyos estudios fueron apoyados mediante la beca 181559 del CONACYT y la beca para estudios de doctorado de la DGEP. El presente estudio también se realizó con el apoyo del subsidio número 35011-H otorgado por el CONACYT al segundo autor. Dirigir correspondencia a los autores a: Laboratorio de Condicionamiento Operante, Facultad de Psicología, UNAM; Ave. Universidad 3004, Cd. Universitaria, México D. F. 04510. México (Correo electrónico: cbruner@servidor.unam.mx o chlg78@yahoo.com). 
la tasa de respuesta fue más alta en los componentes de reforzamiento con agua que en los componentes de extinción cuando se mantuvieron en efecto los programas múltiples. Estos resultados muestran que el BIP se puede controlar mediante un procedimiento de discriminación operante y apoyan la idea de que el BIP se puede explicar conforme a los principios conocidos del condicionamiento operante.

Palabras clave: beber inducido por el programa, reforzamiento con agua, discriminación operante, presiones a una palanca, ratas.

\begin{abstract}
Previous attempts to condition schedule-induced drinking (SID) to formerly neutral stimuli have involved Pavlovian-conditioning procedures. Such strategy has yielded mixed results. According to the idea that SID may reduce to the operant conditioning of the water-producing response with water as its reinforcer, the establishment of stimulus control on SID was attempted using an operant-discrimination procedure. Three food-deprived rats were exposed to mixed and multiple schedules of water-reinforcement. In $32 \mathrm{~s}$ reinforcement components, lever pressing produced water according to a random-interval $6 \mathrm{~s}$ schedule. In $64 \mathrm{~s}$ extinction components, lever pressing did not produce water reinforcement. Food pellets were delivered concurrently according to a random-time $60 \mathrm{~s}$ schedule. For all rats, the rate of lever pressing was similar in both components when the mixed schedules were in effect. When the multiple schedules were in effect response rates were higher in the waterreinforcement components than in the extinction components. These results show that SID can be subjected to an operant discrimination and support the idea that SID reduces to the known principles of operant conditioning.

Key words: schedule-induced drinking, water reinforcement, operant discrimination, lever pressing, rats.
\end{abstract}

Además de controlar la conducta reforzada, los programas de reforzamiento pueden propiciar la ocurrencia de conductas no relacionadas directamente con la obtención del reforzador, como por ejemplo roer un trozo de madera, correr en una rueda o beber agua. Se ha denominado a estas conductas como conductas inducidas por el programa de reforzamiento, de las cuales el beber inducido por el programa (BIP) ha sido considerado el prototipo experimental de estas conductas (Staddon, 1977). EI BIP consiste en que al exponer a ratas privadas de comida a un programa de entrega intermitente de comida, las ratas beben cantidades sustanciales de agua si se encuentra disponible una fuente de agua en la cámara experimental. Lo sorprendente 
del procedimiento de BIP es que puede ocasionar que ratas sin privación de agua consuman en tan solo tres horas hasta cuatro veces su consumo diario de agua bajo alimentación libre (Falk, 1961a).

Con el propósito de explicar el establecimiento y el mantenimiento del BIP algunos investigadores propusieron que el beber agua era una conducta operante reforzada accidentalmente por la entrega de la comida (Clark, 1962; Falk, 1961b; Segal, 1965). La hipótesis del condicionamiento supersticioso del BIP no prosperó debido a que se mostró que el consumo de agua se desarrolló consistentemente bajo un procedimiento en el que se programó una demora reiniciable entre el consumo de agua y la entrega de la siguiente comida (Falk, 1964).

En otro intento por explicar el BIP se propuso que el beber agua era una conducta respondiente evocada por la comida (Wetherington, 1982). Para probar esta hipótesis, en varios estudios se intentó determinar el control de un estímulo neutral sobre el BIP mediante procedimientos de condicionamiento Pavloviano. En estos estudios se presentaron aisladamente estímulos asociados previamente a la comida, para determinar si los estímulos podían evocar el BIP (Porter \& Hamm, 1984). El control del estímulo sobre el BIP no fue consistente entre diferentes estudios. Mientras que en algunos estudios se reportó que las presentaciones aisladas del estímulo controlaron un aumento del consumo de agua, en otros estudios se reportó que las presentaciones aisladas del estímulo controlaron una disminución del consumo de agua (Allen \& Porter, 1977; Jacquet, 1972; Rosenblith, 1970; Stone, Lyon, \& Anger, 1978).

Los fracasos en los intentos por establecer claramente el control del estímulo sobre el BIP con procedimientos de condicionamiento Pavloviano, así como los resultados contradictorios acerca del condicionamiento operante del BIP, se han usado como evidencia a favor de la idea de que el BIP constituye una tercera categoría conductual diferente de la operante y de la respondiente (Falk, 1971; Staddon, 1977). Por ejemplo, para Staddon el BIP pertenece a la categoría de las conductas interinas las cuales son esencialmente diferentes de la conducta operante y respondiente. De acuerdo con Staddon, las conductas interinas se caracterizan por ocurrir con mayor frecuencia al inicio del intervalo entre entregas de comida sucesivas, esto es, durante un periodo de tiempo en el que es poco probable recibir una siguiente comida. Es notable que durante los últimos 45 años se continuó considerando al BIP como un fenómeno inexplicable conforme a los principios en la teoría de la conducta (e.g., De Carolis, 2003; López, López, Sánchez, \& Flores, 2006).

Tratar de explicar al BIP conforme a una nueva categoría conductual representa un serio problema para el análisis experimental de la conducta debido a que le resta generalidad a los principios establecidos en la teoría de la conducta (Skinner, 1938). La inclusión de nuevos conceptos en la teoría 
de la conducta, como proponer una tercera clase de conducta para explicar el BIP, puede representar un retroceso en esta disciplina cuando estos constructos no representan categorías legitimas y justificables de análisis (Cabrer, Daza \& Ribes, 1975). De acuerdo con Cabrer et al., la introducción de nuevos conceptos o la reformulación de los ya existentes sólo se justifica cuando la validez de los datos que propician la revisión de los principios no es objetable y cuando no es posible analizar los datos como fenómenos locales o casos limítrofes de una ley o principio.

Debido a que en los procedimientos de BIP las ratas se encuentran explícitamente privadas de comida, en los intentos anteriores por tratar de condicionar el BIP con procedimientos operantes (e.g., Clark, 1962) y Pavlovianos (e.g., Rosenblith, 1970), se sugirió que la comida es el reforzador de la conducta de beber agua o que la comida es el estímulo incondicionado que provocaba el beber. A diferencia de la hipótesis de que la comida es el reforzador del beber, Bruner y Avila (2002) dedujeron que la conducta de producir agua, como lamer un tubo o presionar una palanca, era reforzada directamente con la obtención de agua. Los autores sugirieron que en los experimentos sobre BIP, la operación de privar de comida a las ratas producía una privación de agua inadvertida en la caja habitación y que durante las sesiones experimentales la entrega de comida a intervalos de tiempo restablecía el consumo de agua. También sugirieron que la función en $U$ invertida del consumo de agua dentro de un intervalo entre comidas constante, que se consideró como una de las características distintivas del BIP que apoyó la propuesta de que el fenómeno constituía una tercera clase de conducta, se debía a que en la mayoría de los experimentos sobre BIP se ha utilizado un programa de reforzamiento con agua de razón fija (RF) 1 . Conforme al RF 1 en los estudios sobre BIP cada lengüetazo al tubo con agua, presión a una palanca o presión a un botón resultó en la entrega de una gota de agua (e.g., Falk, 1961a; Heyman \& Bouzas, 1980). Una implicación de la hipótesis de Bruner y Avila es que si en los procedimientos de BIP el agua es el reforzador de la conducta que produce el agua, entonces se puede emplear cualquier programa de reforzamiento para entregar el agua contingente a la respuesta que la produce. En su estudio, Bruner y Avila reforzaron intermitentemente con una gota de agua las presiones a una palanca conforme a programas de intervalo fijo (IF) y concurrentemente entregaron comida conforme a un programa de tiempo al azar (TA). Encontraron que la respuesta de presión a la palanca por agua se estableció y mantuvo en las tres ratas con un diferente valor del IF para cada rata. También encontraron para las tres ratas que la tasa de respuesta dentro del intervalo entre entregas de agua se distribuyó de forma semejante a un festón típico de IF (Dews, 1970). En la ultima fase de su estudio, manteniendo constante el programa de IF por agua, los autores suspendieron el programa de entrega de comida. Esta manipulación resultó en una dismi- 
nución de la tasa de respuesta para obtener agua, lo cual es un resultado típico de los estudios sobre BIP (Falk, 1971). De acuerdo con los autores este resultado mostró que la presencia de comida es una condición necesaria para establecer al agua como reforzador. Mediante su estudio, Bruner y Avila demostraron que la función bitónica de consumo de agua no es distintiva del BIP y que es posible el condicionamiento operante de la conducta de beber mediante el reforzamiento intermitente con agua de la conducta que produce el agua en una situación de BIP, i.e. en ratas privadas de comida y durante la entrega de comida a intervalos de tiempo.

Dado que Bruner y Avila (2002) mostraron que es posible controlar el BIP mediante procedimientos de condicionamiento operante, entonces en principio es posible replicar diversos fenómenos del condicionamiento operante como determinar el control que un estímulo puede ejercer sobre la respuesta para obtener agua en un procedimiento de BIP. En el condicionamiento operante se han desarrollado diferentes procedimientos que se han empleado en el estudio del control de un estímulo neutral sobre una respuesta (Terrace, 1966). En la investigación sobre el control de estímulos con procedimientos de condicionamiento operante, una de las funciones de un estímulo sobre la conducta que más se ha investigado es la función discriminativa (Keller \& Schoenfeld, 1950). En gran parte de los estudios sobre el establecimiento de una discriminación operante se han empleado programas múltiples debido a que este tipo de programa permite probar sistemáticamente diferentes parámetros que afectan la discriminación (Williams, 1983). Los programas múltiples se componen de dos o más programas de reforzamiento independientes entre sí que se presentan sucesivamente, cada uno de ellos correlacionado con un estímulo exteroceptivo diferente (Ferster \& Skinner, 1957). Cuando se programan diferentes contingencias de reforzamiento en los componentes de un programa múltiple se puede observar la modulación de la tasa y del patrón de respuesta conforme a las contingencias de reforzamiento programadas en cada componente (Nevin \& Grace, 2000).

El procedimiento que se utilizó en los estudios previos para tratar de determinar el control del estímulo sobre el BIP en general consistió en presentar la comida y el estímulo intermitentemente durante las sesiones, mientras que el agua se encontraba continuamente disponible (Wetherington, 1982). Si en los procedimientos de BIP el agua es el reforzador de la conducta que produce el agua como sugirieron Bruner y Avila (2002), los procedimientos de los estudios en los que se trató de determinar el control del estímulo sobre el BIP pueden ser clasificados operacionalmente como procedimientos de condicionamiento Pavlovianos debido a que el reforzador de agua se presentó durante el estímulo y en ausencia del estímulo independientemente de la conducta de los sujetos (Rescorla, 1967). Es posible que la falta de consistencia entre los resultados de los estudios previos acerca del control del estímulo sobre el 
BIP se deba a que en estos estudios se utilizaron procedimientos de condicionamiento Pavloviano. Considerando los hallazgos de Bruner y Avila sobre el BIP, posiblemente es necesario utilizar procedimientos de condicionamiento operante para determinar el control del estímulo sobre el BIP, lo cual implicaría entregar el reforzador de agua en presencia del estímulo solamente si ocurre una respuesta y suspender la entrega de agua en ausencia del estímulo. El reforzamiento diferencial de la respuesta que produce el agua en presencia del estímulo, debería dotar a dicho estímulo con control sobre el beber agua. Por lo tanto, el propósito del presente estudio fue probar si era posible establecer una discriminación operante en el caso del BIP utilizando un programa múltiple de dos componentes, con el que se reforzó con agua a la respuesta que produce el agua en el componente de reforzamiento y se suspendió el reforzamiento con agua en el componente de extinción. Para mantener presentes las condiciones del BIP durante las sesiones experimentales se entregó comida independientemente de la conducta de los sujetos y se utilizó a ratas privadas únicamente de comida.

Como un control de los efectos de la presentación de los estímulos en los componentes del programa múltiple sobre la tasa de la respuesta, en el presente estudio se suspendió la presentación de los estímulos en diferentes bloques de sesiones, con lo cual se convirtió al programa múltiple en un mixto (Nevin, 1973).

\section{MÉTODO}

\section{Sujetos}

Se utilizaron tres ratas Wistar macho de tres meses de edad, experimentalmente ingenuas. Se controló la cantidad de alimento entregado a las ratas en las cajas habitación para mantenerlas al $80 \%$ de su peso ad libitum. Las ratas tuvieron acceso continuo al agua en las cajas habitación.

\section{Aparatos}

Se utilizaron tres cajas experimentales Med Associates Inc. (ENV-007). En cada caja se instaló una palanca (ENV-110RM) sensible a una fuerza de 0.15 $\mathrm{N}$, que se ubicó al centro del panel frontal y a $7 \mathrm{~cm}$ del piso de las cajas. A la izquierda de la palanca y a $1 \mathrm{~cm}$ del piso de las cajas se instaló un recipiente para el agua (ENV-200R3M) conectado mediante una manguera a una válvula solenoide (ENV-201A) colocada en la parte posterior del panel frontal. La válvula entregó gotas de agua de $0.1 \mathrm{~mL}$. A la derecha de la palanca y a $1 \mathrm{~cm}$ del piso de las cajas se instaló un recipiente para la comida (ENV-200R1AM) conectado mediante una manguera a un dispensador de bolitas de comida (ENV-203IR) ubicado en la parte posterior del panel frontal. Se utilizaron bo- 
litas de comida de $25 \mathrm{mg}$, que se fabricaron remoldeando polvo de comida para ratas (Harland Teklad). En el panel frontal también se colocó un sonalert (ENV-223AM) ubicado $25 \mathrm{~cm}$ por arriba de la palanca y dos focos de $28 \mathrm{~V}$ (ENV-221M), uno ubicado $6 \mathrm{~cm}$ por arriba de la palanca y el otro ubicado 11 $\mathrm{cm}$ por arriba del recipiente para la comida. El sonalert produjo un tono de 2.9 $\mathrm{kHz}$ y $75 \mathrm{~dB}$. Al centro del panel posterior de cada caja y a $25.5 \mathrm{~cm}$ del piso se instaló un foco de $28 \mathrm{~V}(\mathrm{ENV}-215 \mathrm{M})$ que proporcionó iluminación continua durante las sesiones. En el panel posterior también se colocó un generador de ruido blanco (ANL-914A) ubicado a la derecha del foco y a $19.5 \mathrm{~cm}$ del piso de las cajas, el cual ayudó a enmascarar sonidos ajenos al experimento. Se introdujo a cada caja experimental dentro de una caja sonoamortiguada (ENV-018) equipada con un ventilador (ENV-025FAC) que facilitó la circulación del aire. Desde una habitación contigua a la habitación en la cual se ubicaron las cajas experimentales, se controlaron y registraron los eventos experimentales mediante una computadora con software Med-PC versión IV, acoplada a una interfase Med Associates Inc.(SG-503).

\section{Procedimiento}

Dado que la adquisición de nuevas respuestas sin la intervención directa del experimentador es un fenómeno robusto (Lattal \& Williams, 1997), no se entrenó a las ratas para acercarse al recipiente para el agua o la comida y tampoco se moldeó la respuesta de presión a la palanca. Se expuso directamente a las tres ratas a 15 sesiones en las que se reforzó con $0.1 \mathrm{~mL}$ de agua cada presión a la palanca (RF 1) y concurrentemente se entregó comida conforme a un programa de tiempo al azar (TA) $60 \mathrm{~s}(t=6, p=.1)$. El programa de TA $60 \mathrm{~s}$ por comida se mantuvo constante durante el resto de las sesiones del experimento. Durante las siguientes cinco sesiones se reforzaron con agua las presiones a la palanca conforme a un programa de intervalo al azar (IA) $6 \mathrm{~s}(t=2 \mathrm{~s}, p=.33)$. Las primeras 20 sesiones tuvieron una duración de una hora ó 50 entregas de agua, lo que ocurriera primero. Posteriormente, se expuso a las ratas durante 30 sesiones a un programa mixto IA $6 \mathrm{~s}$ por agua extinción (mixto IA $6 \mathrm{~s}$ Ext). Los componentes de IA tuvieron una duración de $32 \mathrm{~s}$ y los componentes de extinción tuvieron una duración de $64 \mathrm{~s}$. Ambos componentes se alternaron al azar, con la restricción de que no ocurrieran sucesivamente más de dos componentes de un mismo tipo. En las siguientes 30 sesiones se cambió el programa mixto por un programa múltiple de reforzamiento con agua (múltiple IA $6 \mathrm{~s}$ Ext). Se señalaron los componentes de IA con el encendido y apagado cada segundo del sonalert y de los dos focos ubicados en el panel frontal y los componentes de extinción se señalaron manteniendo encendidos tanto el sonalert como los dos focos. En 30 sesiones más se restableció el programa mixto IA 6 s Ext y durante otras 30 sesiones adicionales se restableció el programa múltiple IA 6 s Ext. Tanto las 
sesiones en las que estuvo en efecto el programa mixto como las sesiones con el programa múltiple terminaron después de que se presentaron $30 \mathrm{com}$ ponentes de reforzamiento y 30 componentes de extinción.

En el presente estudio se utilizó un programa de entrega de comida de TA 60 s debido a que en estudios anteriores sobre BIP se ha observado que facilita el desarrollo del consumo de agua (e.g., Bruner \& Avila, 2002). El valor del programa de reforzamiento de IA y la duración de los componentes de los programas mixtos y múltiples se eligieron debido a que en experimentos anteriores realizados en nuestro laboratorio se observó que estos valores controlan una clara separación de las tasas de respuesta en los componentes de reforzamiento y de extinción (e.g., Escobar \& Bruner, 2002).

\section{RESULTADOS}

En las primeras 15 sesiones en las que estuvo en efecto el programa de RF 1 con agua se encontró que el número de reforzadores de agua obtenidos por las ratas aumentó gradualmente hasta que en las últimas sesiones las tres ratas obtuvieron los 50 reforzadores disponibles en cada sesión. Durante las cinco sesiones en las que se entregó el agua conforme al IA $6 \mathrm{~s}$ las tres ratas obtuvieron las 50 entregas de agua programadas en cada sesión.

En la literatura sobre discriminación operante, la tasa de respuesta ha sido una de las principales variables dependientes con la cual se ha medido el efecto de un procedimiento de discriminación en la conducta (Dinsmoor, 1995). En la Figura 1 se presenta para cada rata la tasa media de respuesta en cada sesión durante los componentes de reforzamiento y de extinción. Este dato se presenta para las condiciones con los programas mixtos y múltiples. Las líneas verticales indican el cambio de cada condición. Durante las condiciones con el programa mixto se encontró que la tasa de respuesta de las tres ratas durante los componentes de reforzamiento fue similar a la tasa de respuesta en los componentes de extinción. En las condiciones en las que estuvo en efecto el programa múltiple se encontró para las tres ratas, que la tasa de respuesta durante los componentes de reforzamiento fue más alta en comparación con la tasa de respuesta durante los componentes de extinción.

El índice de discriminación de la tasa de respuesta es un análisis reportado frecuentemente en estudios en los que se utilizan programas múltiples debido a que permite observar el control de los estímulos sobre la tasa de respuesta (Nevin, 1973). En la Figura 2 se presenta para las tres ratas el índice de discriminación de la tasa de respuesta en función del paso de las sesiones de exposición a los programas mixtos y múltiples. El índice de discriminación se calculó dividiendo la tasa de respuesta obtenida durante los componentes 


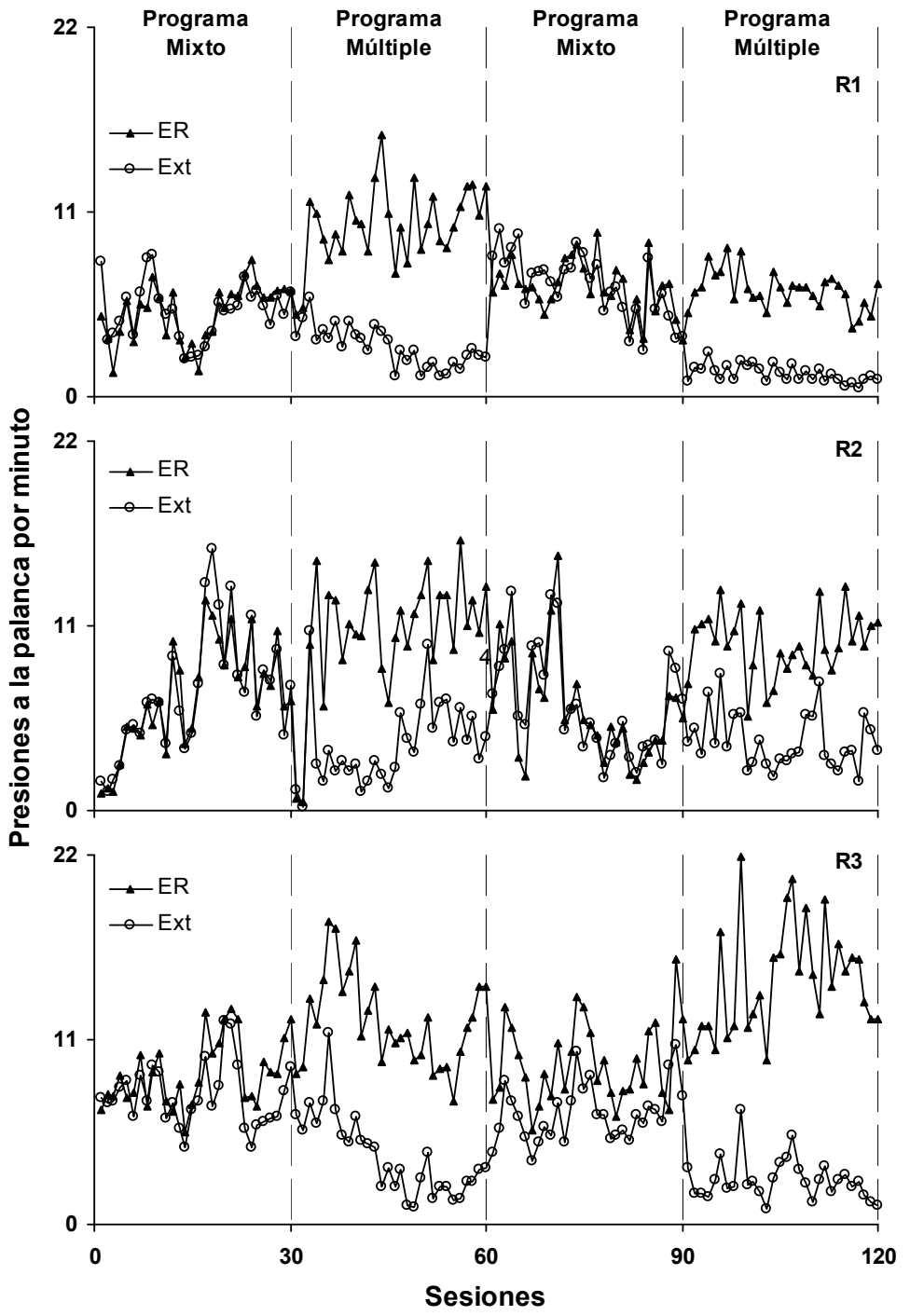

Figura 1. Tasa media de respuesta en cada sesión en los componentes de reforzamiento (ER) y de extinción (EXT), durante la presentación de los programas mixtos y múltiples. Las líneas verticales indican el cambio de cada condición. 

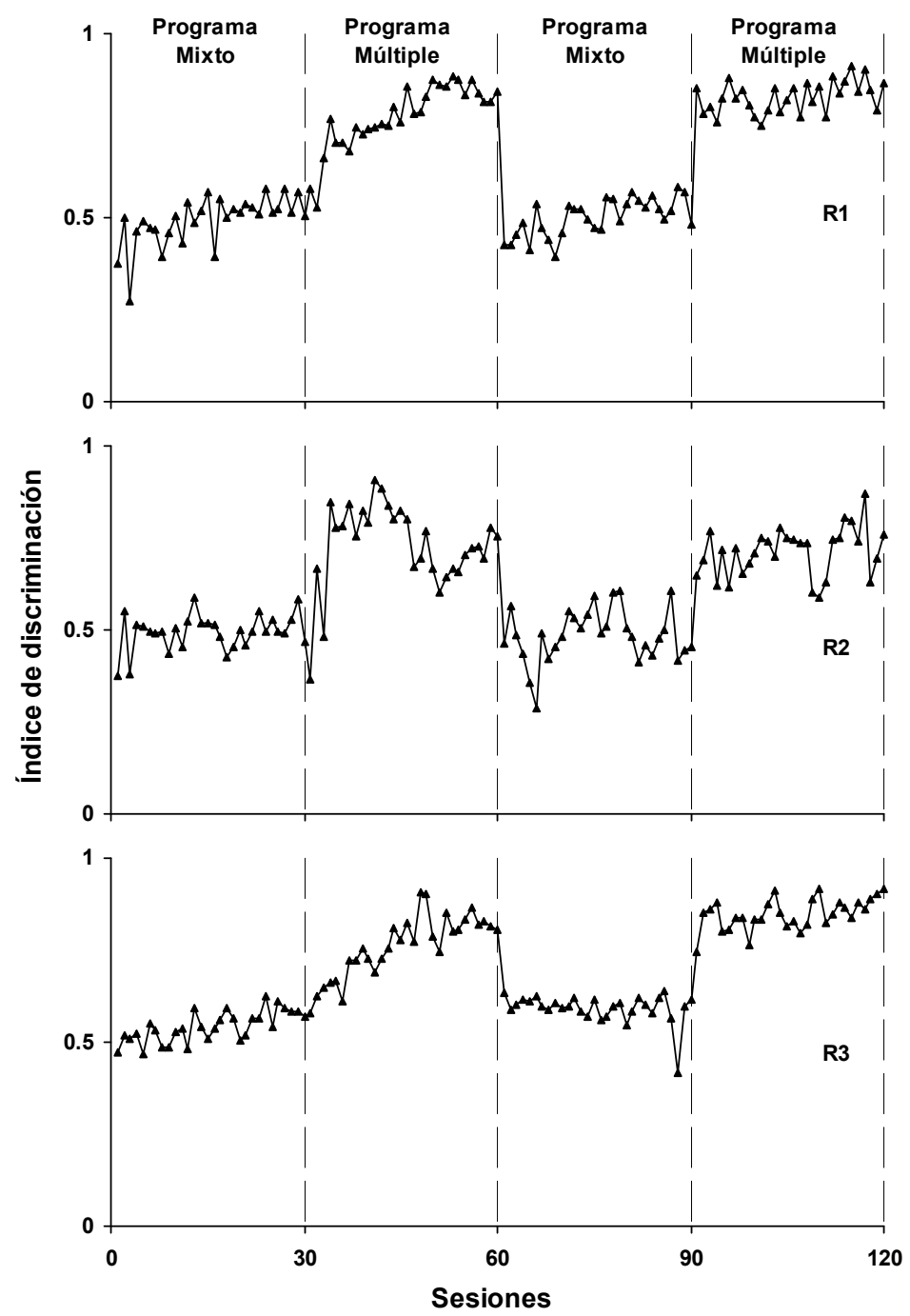

Figura 2. Índice de discriminación de la tasa de respuesta en cada sesión durante la presentación de los programas mixtos y múltiples. Las líneas verticales indican el cambio de cada condición. 
de reforzamiento entre la suma de la tasa de respuesta obtenida durante los componentes de reforzamiento y de extinción (Dinsmoor, 1952). Durante las sesiones en las que estuvo en efecto el programa mixto se encontró que el índice de discriminación medio de las tres ratas fue de aproximadamente 0.5 . Respecto a las condiciones con el programa mixto, cuando se mantuvo en efecto el programa múltiple se encontró que el índice de discriminación medio de las tres ratas aumentó a 0.8, aproximadamente.

Para determinar si el aumento en la tasa media de respuesta en el componente de reforzamiento ocasionado por cambiar el programa mixto por un múltiple, se relacionó con un cambio en la tasa media de reforzamiento en la Figura 3 se muestra para los tres sujetos la tasa media de reforzamiento con agua obtenida en cada sesión, durante la presentación de los programas mixtos y múltiples. Las líneas verticales indican el cambio de cada condición. Se encontró que cambiar el programa mixto por un múltiple no controló algún cambio sistemático en la tasa media de reforzamiento con agua para los tres sujetos.

En algunos estudios con procedimientos de discriminación operante se reportó que durante el establecimiento de la discriminación, la latencia entre el inicio del ED y la ocurrencia de una respuesta disminuye progresivamente, mientras que la latencia de la primer respuesta después del inicio del E $\Delta$ aumenta (e.g., Dinsmoor, 1952). En la Figura 4 se muestra por separado la latencia media en cada sesión de la primera respuesta después del inicio de un componente de reforzamiento o uno de extinción, durante la presentación de los programas mixtos y múltiples. Las líneas verticales indican el cambio de cada condición. En las sesiones en las que se reforzó con agua el palanqueo conforme al programa mixto se encontró que la latencia obtenida durante los componentes de reforzamiento fue similar a la latencia obtenida durante los componentes de extinción. En las sesiones en las que se implementó el programa múltiple se encontró que la latencia fue más larga durante los componentes de extinción que durante los componentes de reforzamiento. 


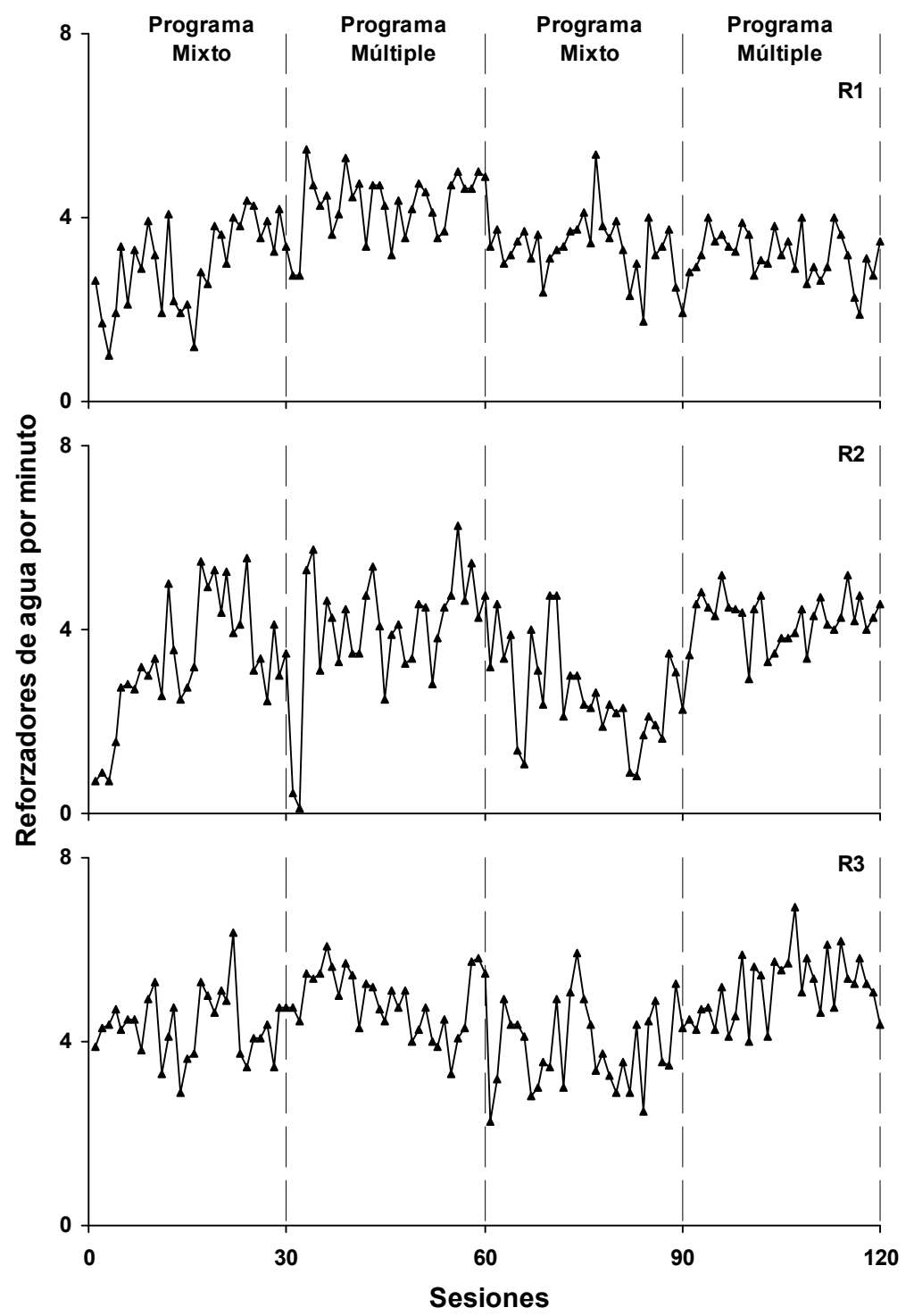

Figura 3. Tasa media de reforzamiento con agua obtenida en cada sesión durante la presentación de los programas mixtos y múltiples. Las líneas verticales indican el cambio de cada condición. 

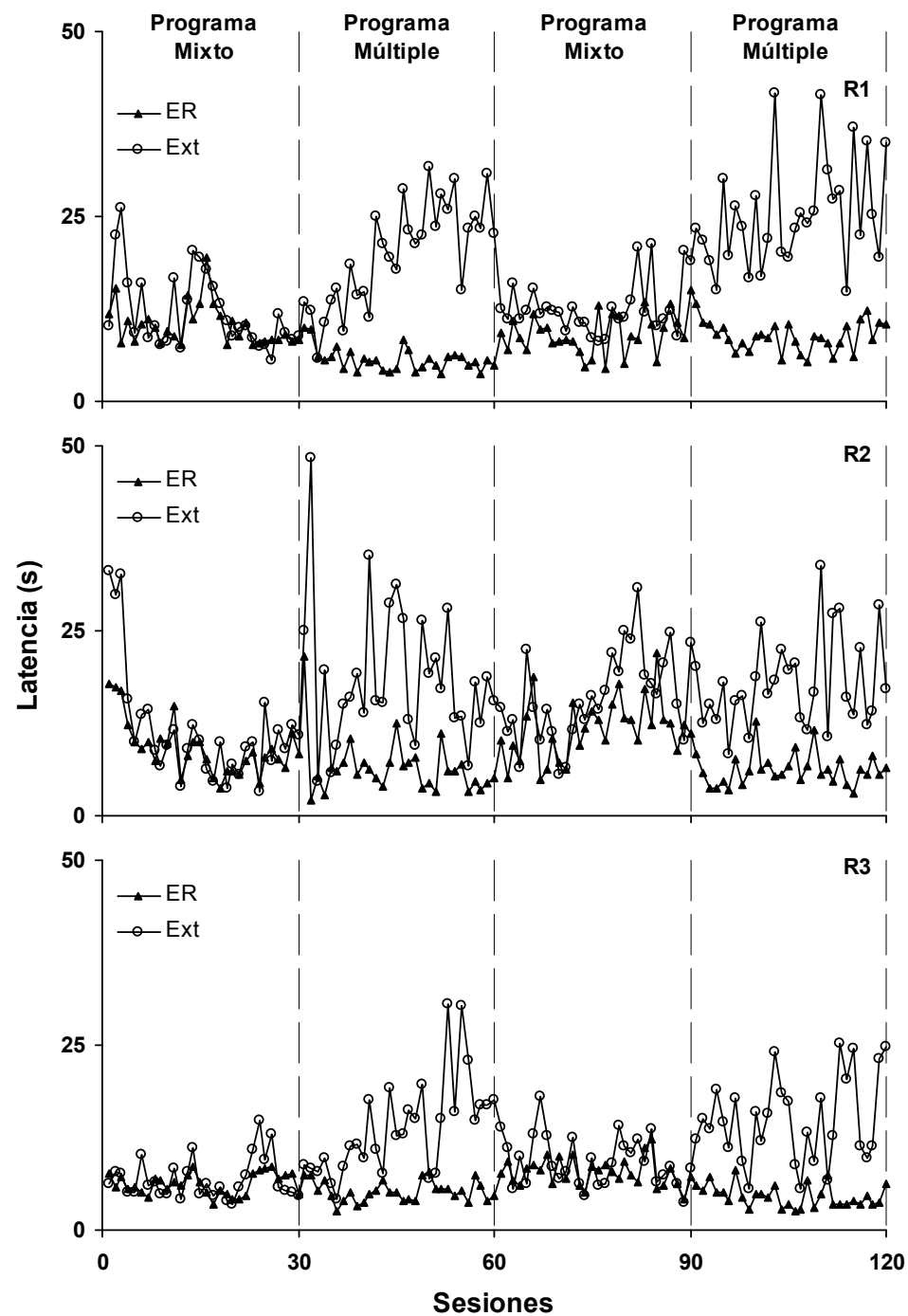

Figura 4. Latencia media de la primera respuesta después del inicio de un componente de reforzamiento (ER) o uno de extinción (EXT) en cada sesión, durante la presentación de los programas mixtos y múltiples. Las líneas verticales indican el cambio de cada condición. 


\section{DISCUSIÓN}

Hasta ahora no había sido posible establecer con claridad las condiciones bajo las cuales es posible aumentar la probabilidad de que ocurra el beber en presencia de un estímulo previamente neutral en un procedimiento de BIP (cf., Porter \& Hamm, 1984). A diferencia de estudios previos sobre BIP (e.g., Rosenblith, 1970) en los cuales se utilizaron procedimientos de condicionamiento Pavloviano para presentar independientemente de la conducta de los sujetos al reforzador de agua en presencia de los estímulos, en el presente estudio se utilizó un procedimiento de condicionamiento operante para presentar los estímulos y el reforzador de agua. Mediante este procedimiento de discriminación operante únicamente se entregó el reforzador de agua en presencia del ED si ocurría una respuesta y se suspendió la entrega del reforzador de agua durante el $\mathrm{E} \Delta$, en un contexto de entrega gratuita de comida. Se encontró que al presentar los programas múltiples la tasa media de respuesta fue mucho más alta en los componentes de reforzamiento que en los componentes de extinción, lo cual indica el establecimiento del control discriminativo de los estímulos sobre la respuesta que produce el agua. En contraste, cuando se presentó el programa mixto se encontró que las ratas presionaron la palanca con tasas semejantes en los componentes de reforzamiento y de extinción, lo cual indica que la separación entre las tasas de respuesta durante la presentación de los programas múltiples se debió a la presentación de los estímulos discriminativos ya que la presentación de estos estímulos fue la única diferencia de procedimiento entre los programas mixtos y múltiples. Estos resultados constituyen la primera demostración clara del control del beber agua, mediante estímulos previamente neutrales en un procedimiento de BIP.

Conforme a los estudios clásicos sobre el establecimiento de una discriminación operante (e.g., Skinner, 1938), en el presente estudio se esperaba que respecto a las condiciones con el programa mixto, al presentar el programa múltiple la tasa de respuesta en presencia del E $\Delta$ disminuyera debido a que en presencia de este estímulo no se reforzó a la respuesta, mientras que se esperaba que la tasa de respuesta en presencia del ED se mantuviera sin cambios debido a que no se varió la frecuencia de reforzamiento programada durante este estímulo. Sin embargo, es sorprendente que además de la disminución de la tasa de respuesta que se esperaba en presencia del E $\Delta$ se observó un aumento de la tasa de respuesta en presencia del ED. Este hallazgo es sorprendente debido a que se asemeja a un fenómeno conocido contraste conductual positivo, el cual generalmente ocurre como resultado de variar la frecuencia de reforzamiento programada en los componentes de un programa múltiple. Por ejemplo, relativo a un programa de múltiple que genera tasas similares de respuesta en los dos componentes del programa 
(e.g., múltiple IV 2 min IV 2 min), al presentar un programa múltiple en el que alternan un componente de reforzamiento y un componente de extinción (e.g., múltiple IV 2 min Ext), la tasa de respuesta en el componente de reforzamiento aumenta mientras que la tasa de respuesta en el componente de extinción disminuye (Catania, 1961; Bloomfield, 1966; Reynolds, 1961, 1963). A pesar de que no es el hallazgo más común, se han reportado efectos de contraste conductual positivo sin variar la frecuencia de reforzamiento programada entre fases experimentales. En estos estudios observó contraste conductual positivo por ejemplo al cambiar un programa múltiple IV 2 min IV 2 min por un múltiple IV 2 min IF 2 min (e.g., Wilkie, 1977) o al cambiar un programa mixto IV 3 min Ext por un múltiple IV 3 min Ext (e.g., Nevin, 1973). Por lo tanto, los cambios en las tasas de la respuesta en presencia del ED y el E $\Delta$ observadas en el presente estudio son semejantes a las interacciones conductuales observadas en estudios sobre condicionamiento operante con programas múltiples de dos componentes en los cuales el sujeto se encuentra privado del estímulo que posteriormente se utiliza como reforzador (cf. Williams, 1983).

Se ha argumentado que con frecuencias de reforzamiento relativamente altas, la tasa de respuesta puede no ser una medida clara de los efectos de un procedimiento de discriminación operante sobre la conducta debido a que durante la presentación del ED la entrega del reforzador puede funcionar como una señal de una alta disponibilidad de reforzamiento (Nevin, 1973). Diferentes investigadores han afirmado que la latencia es una medida de la tendencia a responder en presencia de los estímulos discriminativos que no esta contaminada por el efecto de la presentación del reforzador (Blough \& Lipsitt, 1971). En el presente estudio, durante la presentación de los programas múltiples, se observó que la latencia de la primera respuesta después del inicio del ED fue más corta que la latencia de la primera respuesta en presencia del E $\Delta$. En cambio, durante la presentación de los programas mixtos la latencia de la primer respuesta durante los componentes de reforzamiento fue similar a la latencia de la primer respuesta en los componentes de extinción. Las diferencias observadas en la latencia de la primera respuesta en presencia de los dos componentes del programa múltiple son consistentes con una amplia literatura acerca de los efectos de procedimientos de discriminación operante sobre la conducta (e.g. Dinsmoor, 1995; Terrace, 1966). Durante la presentación de los programas múltiples es claro que reforzar con agua a la respuesta que la produce en presencia del ED fue la condición crucial en la formación de la discriminación.

Tanto los investigadores que apoyaron la explicación del condicionamiento Pavloviano, como los que apoyaron la explicación del condicionamiento supersticioso del BIP, enfatizaron la función de la comida en los procedimientos de BIP al afirmar que la comida reforzaba adventiciamente la conducta de 
beber agua o al proponer que la comida era el estímulo incondicionado que provocaba el beber (e.g., Clark, 1962; Stone, Lyon, \& Anger, 1978). Conforme a estas dos explicaciones sobre el BIP se hubiera predicho en el presente estudio que la tasa de respuesta en los componentes de reforzamiento sería similar a la tasa de respuesta en los componentes de extinción, dado que la frecuencia con la que se presentó el "estímulo incondicionado" o el "reforzador adventicio" del beber fue similar en los componentes de reforzamiento y de extinción tanto para los programas mixtos como múltiples. Esta predicción podría parecer cierta para el caso del programa mixto en donde a pesar de que no había disponibilidad de agua durante los componentes de extinción, la tasa de respuesta en este componente fue similar a la tasa de respuesta en el componente de reforzamiento. Sin embargo, durante el programa múltiple se observaron tasas diferenciales de respuesta en los componentes de reforzamiento y de extinción, a pesar de que la frecuencia con la que se presentó la comida (i.e., el supuesto "estímulo incondicionado" o "reforzador adventicio" del BIP) fue similar en ambos componentes del programa. A diferencia de la explicación del condicionamiento Pavloviano y del condicionamiento supersticioso del BIP, los datos del presente estudio pueden ser explicados sin dificultad como un caso de condicionamiento operante en el que se observan los efectos conocidos de los programas mixtos y múltiples sobre la conducta que produce el agua, lo cual cuestiona el papel de la comida como el reforzador adventicio de la conducta de beber agua o como el estímulo incondicionado que provoca el beber.

Los fracasos en los intentos anteriores por tratar de establecer el control de un estímulo neutral sobre el BIP mediante procedimientos de condicionamiento Pavloviano se usaron como parte de la evidencia a favor de la propuesta de que el BIP pertenece a una tercera categoría conductual diferente de las operantes y las respondientes (Falk, 1971; Staddon, 1977). EI BIP continua siendo considerado como un fenómeno anómalo y difícil de explicar conforme al conocimiento establecido en el análisis experimental de la conducta, lo cual ha propiciado la aparición de explicaciones ajenas a los principios de esta disciplina, como por ejemplo la explicación del BIP como producto de la frustración ocasionada por la exposición de los sujetos a la entrega intermitente de comida (De Carolis, 2003; López, López, Sánchez, \& Flores, 2006). Otros autores propusieron que la entrega intermitente de comida establecía una situación de conflicto, por lo que el consumo de agua ocurría como una conducta de desplazamiento (e.g., Falk, 1977). Dado que en el presente estudio se encontró que es posible establecer claramente el control de un estímulo neutral sobre el BIP entregando al agua como reforzador de la respuesta que produce el agua conforme a un procedimiento de condicionamiento operante, los hallazgos del presente estudio aportan 
evidencia a favor de la reducción del BIP como un caso de condicionamiento operante (Bruner \& Avila, 2002).

Otros estudios en los cuales se ha demostrado que el agua es el reforzador de la conducta que la produce en los procedimientos de BIP, son los estudios de Roca y Bruner (2003) y Ruiz y Bruner (2005). Roca y Bruner condujeron un estudio con el propósito de probar si era posible reproducir la conocida correlación entre la tasa de respuesta y la tasa de reforzamiento usando el agua como reforzador en una situación de BIP. Utilizando a ratas privadas de comida como sujetos, Roca y Bruner reforzaron con agua las presiones a una palanca conforme a un programa de reforzamiento de intervalo al azar (IA). En condiciones sucesivas se alargó el IA de 8 a $2222 \mathrm{~s}$. Concurrentemente se entregó comida conforme a programas de tiempo variable durante las sesiones. Los autores reportaron una correlación alta y positiva entre la tasa de presionar la palanca y la tasa obtenida de reforzamiento con agua, lo cual mostró que el BIP era sensible al parámetro de frecuencia de reforzamiento de agua, bien documentado en condicionamiento operante (e.g., Farmer, 1963). Ruiz y Bruner investigaron el efecto de transformar progresivamente un programa de IF de reforzamiento con agua en un procedimiento de BIP sobre los lengüetazos a un tubo, utilizando a ratas como sujetos. Los autores mostraron que cada una de las variables presentes en los procedimientos de BIP (i.e., la privación de comida, la entrega periódica de comida y la entrega continua de agua) tuvieron efectos sobre las respuestas por agua idénticos a los efectos de estas variables reportados previamente en estudios sobre condicionamiento operante y motivación. En conjunto, los datos de Bruner y sus colaboradores y los datos del presente estudio muestran sin ambigüedad que se puede integrar al BIP a los principios establecidos en la teoría de la conducta si tan solo se considera al agua como reforzador de la conducta que la produce en los procedimientos de BIP.

\section{REFERENCIAS}

Allen, J. D., \& Porter, J. H. (1977). Sources of control over schedule-induced drinking produced by second-order schedules of reinforcement. Physiology and Behavior, $18,853-863$.

Bloomfield, T. M. (1966). Two types of behavioral contrast in discrimination learning Journal of the Experimental Analysis of Behavior, 9, 155-161.

Blough, D. S., \& Lipsitt, L. P. (1971). The discriminative control of behavior. In Kling, J. W., \& Riggs, L. A. (Eds.), Woodworth \& Schlosberg's Experimental Psychology (pp. 743-792). New York: Holt, Rinehart and Winston, Inc.

Bruner, C. A., \& Avila, R. (2002). Adquisición y mantenimiento del palanqueo en ratas sin privación explícita del reforzador. Revista Mexicana de Análisis de la Conducta, 28, 107-130. 
Cabrer, F., Daza, B. C., \& Ribes, E. (1975) Teoría de la Conducta: ¿Nuevos conceptos o nuevos parámetros?. Revista Mexicana de Análisis de la Conducta, 1, 191212.

Catania, A. C. (1961). Behavioral contrast in a multiple and concurrent schedule of reinforcement. Journal of the Experimental Analysis of Behavior, 4, 335-342.

Clark, F. C. (1962). Some observations of the adventitious reinforcement of drinking under food reinforcement. Journal of the Experimental Analysis of Behavior, 5, 61-63.

De Carolis, N. A., Myracle, A., Erbach, J., Glowa, J., Flores, P., \& Riley, A. L. (2003). Strain-dependent differences in schedule-induced polydipsia: An assessment in Lewis and Fischer rats. Pharmacology, Biochemestry and Behavior, 74, 755763.

Dews, P. B. (1970). The theory of fixed-interval responding. In: W. N. Schoenfeld (Ed.), The Theory of Reinforcement Schedules, (pp). Englewood Cliffs, NJ: Prentice Hall.

Dinsmoor, J. A. (1952). The effect of hunger on discriminated responding. Journal of Abnormal and Social Psychology, 47, 67-72.

Dinsmoor, J. A. (1995). Tutorial: Stimulus control: Part I. The behavior analyst, 18, 51-68.

Escobar, R., \& Bruner, C. A. (2002). Efectos de la frecuencia de reforzamiento y la duración del componente de extinción en un programa de reforzamiento mixto sobre las respuestas de observación en ratas. Revista Mexicana de Análisis de la Conducta, 28, 41-46.

Falk, J. L. (1961a). Production of polydipsia in normal rats by an intermittent food schedule. Science, 133, 195-196.

Falk, J. L. (1961b). The behavioral regulation of water-electrolyte. In M. R. Jones (Ed.). Nebraska Symposium on Motivation (pp. 12-19). Lincoln, NE: University Press.

Falk, J. L. (1964). Studies on schedule-induced polydipsia. En M. J. Wayner (Ed.), Thirst: First International Symposium on thirst in the regulation of body water (pp. 95-116). New York: Pergamon Press.

Falk, J. L. (1971). The nature and determinants of adjunctive behavior. Physiology and Behavior, 6, 577-588.

Falk, J. L. (1977). The origin and functions of adjunctive behavior. Animal Learning and Behavior, 5, 325-335.

Farmer, J. (1963). Properties of behavior under random interval reinforcement schedules. Journal of the Experimental Analysis of Behavior, 6, 607-616.

Ferster, C. B., \& Skinner, B. F. (1957). Schedules of Reinforcement. Englewood Cliffs, NJ: Prentice-Hall.

Heyman, G. M., \& Bouzas A. (1980). Context dependent changes in the reinforcing strength of schedule-induced drinking. Journal of the Experimental Analysis of Behavior, 33, 327-335.

Jacquet, Y. F. (1972). Schedule-induced licking during multiple schedules. Journal of the Experimental Analysis of Behavior, 17, 413-423.

Keller, F. S., \& Schoenfeld, W. N. (1950). Principles of Psychology. New York: Appleton Century Crofts. 
Lattal, K. A., \& Williams, A. M. (1997).Body weight and response acquisition with delayed reinforcement. Journal of the Experimental Analysis of Behavior, 67, 131143.

López, G. M., López C. G., Sánchez, A. M. C., \& Flores, C. P. (2006). Los efectos de la anfetamina administrada en el córtex prefrontal medial sobre las diferencias individuales en polidipsia inducida por programa. International Journal of Psychology and Psychological Therapy, 6, 261-272.

Nevin, J. A. (1973). Stimulus control. In J. A. Nevin (Ed.), The study of behavior (pp. 115-152). Glenview, IL: Scott, Foresman.

Nevin, J. A., \& Grace, R. C. (2000) Behavioral momentum and the Law of Effect. Behavioral And Brain Sciences, 23, 73-130.

Porter, J. H., \& Hamm, R. J. (1984). Associative control of schedule-induced drinking. Animal Learning and Behavior, 12, 339-340.

Rescorla, R. A. (1967) Pavlovian conditioning and its proper control procedures. Psychological Review, 74, 71-80

Reynolds, G. S. (1961). Relativity of response rate and reinforcement frequency in a multiple schedule. Journal of the Experimental Analysis of Behavior, 4, 179-184.

Reynolds, G. S. (1963). Some limitations on behavioral contrast and induction during successive discrimination. Journal of the Experimental Analysis of Behavior, 6, 131-139.

Roca, A., \& Bruner, C. A. (2003). Efectos de la frecuencia de reforzamiento sobre el palanqueo por agua en ratas privadas de comida. Revista Mexicana de Análisis de la Conducta, 29, 119-130.

Rosenblith, J. Z. (1970). Polydipsia induced in the rat by a second-order schedule. Journal of the Experimental Analysis of Behavior, 12, 139-144.

Ruiz, J. A., \& Bruner, C. A. (2005). Transformación de un programa intervalo fijo de reforzamiento con agua en un procedimiento de beber inducido por el programa. Revista Mexicana de Análisis de la Conducta, 29, 119-130.

Segal, E. F. (1965). The development of water drinking on a dry-food free-reinforcement schedule. Psychonomic Science, 16, 29-30.

Skinner, B. F. (1938). The behavior of organisms. New York: Appleton-CenturyCrofts.

Staddon, J. E. R. (1977). Schedule-induced behavior. En W. K. Honig, \& J. E. R. Staddon (Eds.), Handbook of Operant Behavior (pp. 125-152). Englewood Cliffs, NJ: Prentice-Hall.

Stone, W., Lyon, D. O., \& Anger, D. (1978) Suppression of postpellet licking by a Pavlovian S+. Bulletin of the Psychonomic Society, 12, 117-119.

Terrace, H. S. (1966). Stimulus control. In W. K. Honig (Ed.), Operant Behavior (pp. 271-344). New York: Appleton-Century-Crofts.

Wetherington, C. L. (1982). Is adjuntive behavior a third class of behavior?. Neuroscience and Biobehavioral Reviews, 6, 329-350.

Wilkie, D. M. (1977) Behavioral contrast produced by a signaled decrease in local rate of reinforcement. Learning and Motivation, 8, 182-193.

Williams, B. A. (1983). Another look at contrast in multiple schedules. Journal of the Experimental Analysis of Behavior, 39, 345-384. 